\title{
Endovascular Coil Embolization of Multiple Pulmonary Arteriovenous Malformations in Osler-Weber-Rendu Disease: A Challenging Pursuit
}

\author{
Jerelyn B. Adviento, Gino Rei A. Quizon, Jonas D. del Rosario, \\ Maria Teresa B. Abola, Wilfred G. Dee and Richard Henry P. Tiongco III \\ Section of Cardiology, Department of Medicine, College of Medicine and Philippine General Hospital, University of the Philippines Manila
}

\begin{abstract}
Pulmonary arteriovenous malformations (PAVMs) are a rare disorder with an incidence of 2-3/1,000,000 population. Approximately $70 \%$ of cases are associated with Osler-WeberRendu Disease. This intrapulmonary malformation causes hypoxemia and dyspnea largely attributed to the right to left shunting.

We present a case of a 25-year-old male who was admitted for fever and headaches with chronic history of epistaxis, hemoptysis, cyanosis and clubbing. Central cyanosis and clubbing were evident with hypoxemia of $65 \%$. Heart sounds and peripheral pulses were normal. There was no systolic bruit noted in the lung bases. Multiple telangiectasias were seen in the truncal area and abdomen. Chest x-ray revealed multichambered cardiomegaly with no opacifications. CBC showed erythrocytosis with hematocrits of $0.68-0.78$. Transthoracic echocardiography showed intact interatrial and interventricular septum, with contrast study suggestive of intrapulmonary shunting. CT angiography revealed PAVMs in bilateral lung fields. Pulmonary angiography demonstrated diffuse PAVMS in the left lung with 3 large PAVMs with multiple feeders $>7 \mathrm{~mm}$ and smaller PAVMs in the Right lower lobe. The diagnosis was Multiple Pulmonary Arteriovenous Malformation, Osler-WeberRendu Disease, Brain Abscess Left Temporoparietal area. Craniotomy with brain abscess evacuation was done. Left pneumonectomy or Right lobectomy was considered but was deemed unacceptable due to high morbidity and mortality. Endovascular coil embolization was done on the 3 large fistulas on the Left lobe. Post procedure, his arterial oxygenation
\end{abstract}

Best Moderated Poster, 44th Annual Convention of the Philippine Heart Association - Philippine College of Cardiology, May 29-31, 2013, EDSA Shangri-La Plaza Hotel, Mandaluyong City.

1st Place - Case Report, Oral Presentation, UP-PGH Department of Medicine 2013 Annual Research Forum.

Corresponding author: Jerelyn B. Adviento, MD

Section of Cardiology

Department of Medicine

Philippine General Hospital

University of the Philippines Manila

Taft Avenue, Ermita, Manila 1000 Philippines

Telephone: +6325548400 local 3670

Email: advientojerelyn@yahoo.com improved to $96 \%$. He has no recurrence of hemoptysis, no headaches and with less episodes of shortness of breath 2 months on follow-up. Diagnosing the complex diffuse intrapulmonary malformations requires a high index of suspicion among patients with chronic cyanosis, chronic history of bleeding, and brain abscess. Treatment of this condition and its associated complications remain a big challenge and should be highly individualized.

Key Words: endovascular coil embolization, multiple pulmonary arteriovenous malformations, Osler-Weber-Rendu disease, Hereditary Hemorrhagic Telangiectasia

\section{Introduction}

Pulmonary arteriovenous malformation (PAVM) is a rare disorder with an incidence of 2-3/1,000,000 population. Approximately $70 \%$ of cases are associated with OslerWeber-Rendu Disease or Hereditary Hemorrhagic Telangiectasia. Diagnosing and managing this condition posed a considerable challenge in a patient who came in for recurrent headaches and fever. This paper focused on our clinical approach to how this intrapulmonary malformation was diagnosed and managed.

\section{Case Presentation}

A 25-year-old Filipino male was initially admitted by Neurology service for recurrent headaches and pallor. He was referred to cardiology service for clearance prior to craniotomy for brain abscess. He had chronic history of epistaxis, hemoptysis, cyanosis and clubbing since he was 9years old with no prior consult. He had family history of early uninvestigated cardiac deaths among his siblings. His birth and developmental history were unremarkable.

The patient was thin, pale and cyanotic. His Oxygen saturation at room air was $65 \%$. There was central cyanosis and clubbing. He was normotensive, mildty tachycardic and febrile. Cardiac exam showed no chest deformities, adynamic precordium, apex was not displaced, with no noted murmurs. His peripheral pulses were normal. Auscultation of the chest revealed bronchovesicular breath sounds with no systolic bruit noted in the lung bases. Multiple telangiectasias were found in the abdomen, in the shoulder, and in the left arm (Figure 1). 


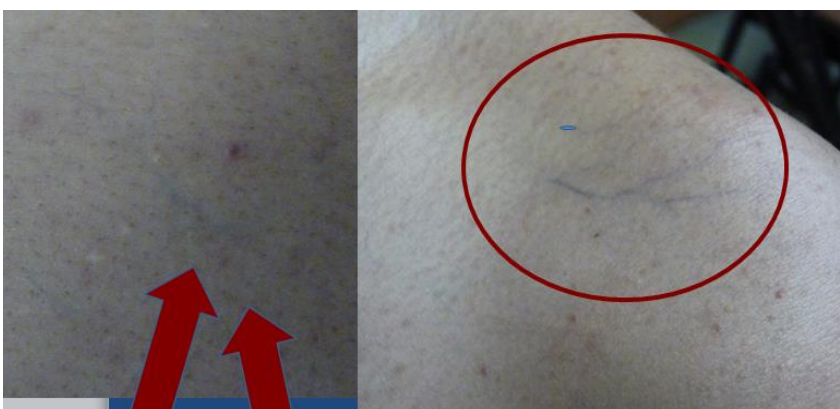

Figure 1. Multiple telangiectasias in the abdomen and shoulder.

His ECG revealed sinus rhythm with normal axis and left ventricular hypertrophy. While his Chest $\mathrm{x}$-ray revealed multi-chambered cardiomegaly with no noted infiltrate, mass and abnormal opacifications (Figure 2). His CBC showed erythrocytosis with hematocrits of 0.68-78. His baseline hematocrit was 0.68 . The arterial blood gas at room air revealed hypoxemia of PO2 40.8 with saturation of $63 \%$. His pulmonary function test was normal. Brain CT scan showed brain abscess in the left temporoparietal lobe.

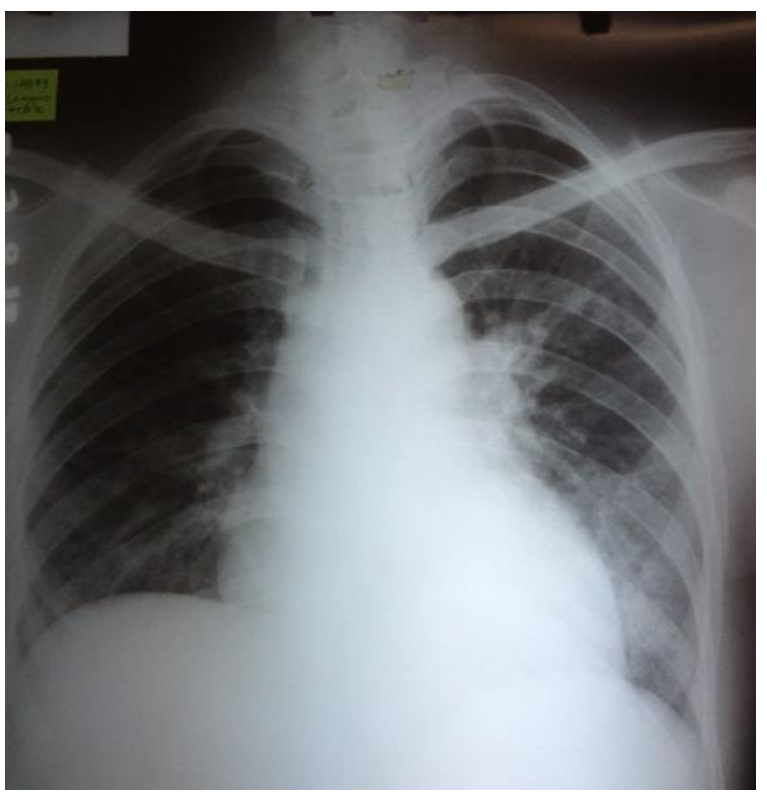

Figure 2. Chest x-ray showing multi-chambered cardiomegaly with no abnormal opacities.

Transthoracic Echocardiogram (TTE) revealed eccentric left ventricular hypertrophy with preserved systolic function, dilated Left atrium with LAVI of 38. Right atrium and right ventricles dimensions were normal. The interatrial and interventricular septums were intact. No stenotic or regurgitation lesion was noted. The pulmonary artery pressure was normal. A suspicious flow was noted in the aortopulmonary area during systole with a consideration for a major aortopulmonary collateral versus venous arterial malformation (Figure 3). Use of Bubble contrast showed a delayed appearance of contrast in the Left atrium after 6 cardiac cycles which suggested an intrapulmonary shunt.

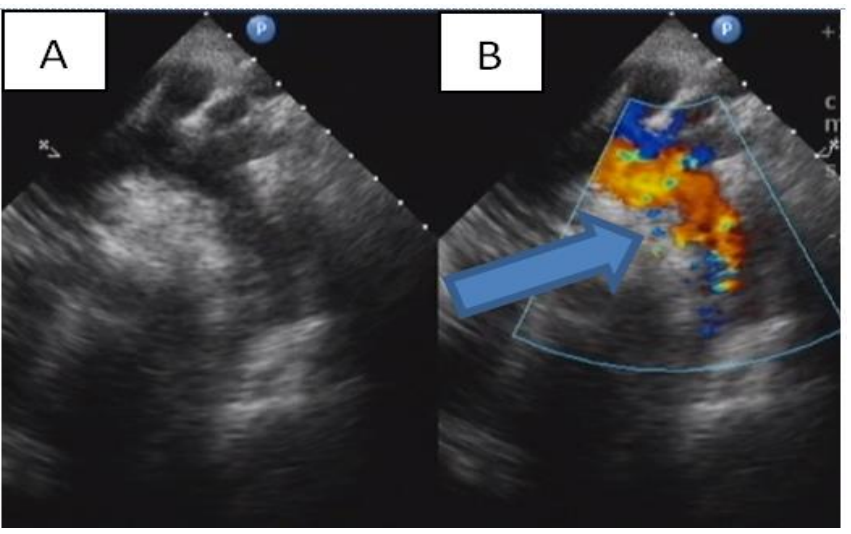

Figure 3. (A) Suprasternal view of the patient's TTE; (B) On application of color revealed a suspicious flow noted in the aortopulmonary area. (Blue arrow)

To document the presence of arteriovenous malformation, a chest CT angiography was done. The study revealed 3 areas of dilated and tortuous tangle of pulmonary arteries and veins were noted (Figure 4a) in the left apicoposterior upper lobe, Left superior segment of lower lobe, Left posterobasal lower lobe and Superior segment of Right lower lobe (Figure 4b).

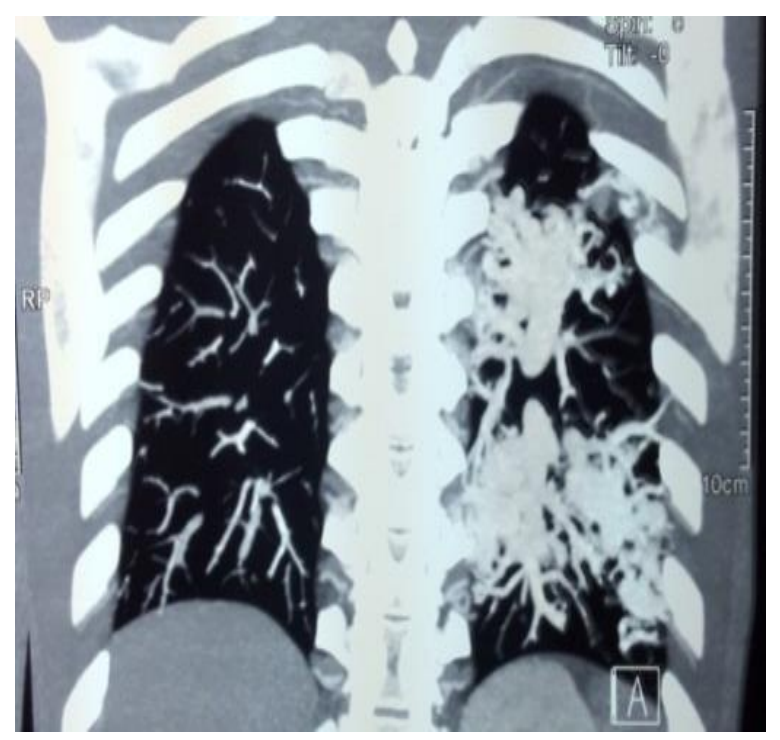

Figure 4a. Coronal cut of Chest CT with contrast which showed 3 large areas of PAVMs 


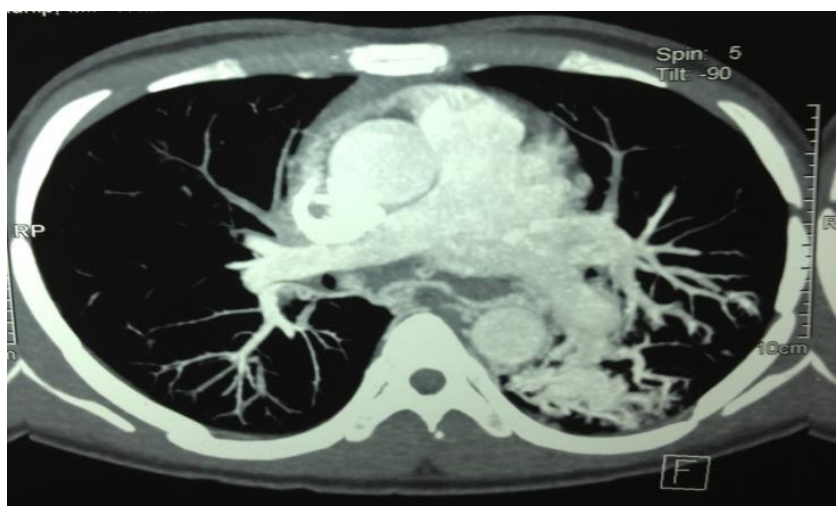

Figure 4 b. Cross sectional view revealed a PAVM in the left posterior basal lobe with multiple feeders.

Pulmonary angiography was done to map the vasculature and locate the feeder arteries. Using Digital Subtotal Angiography (DSA) diffuse PAVMS were found in the left lung field (Figure 5a). Selective catheterization of the left Pulmonary artery showed 3 large PAVMs with feeders as large as $7 \mathrm{~mm}$ were identified (Figure 5b,c). Selective injection of contrast media to the right pulmonary artery demonstrated smaller PAVMs and fistulas. Resting hemodynamics showed elevated RV systolic pressures with normal RA, PA and LV pressures. Oximetry run was attempted but his saturations were undetectable.

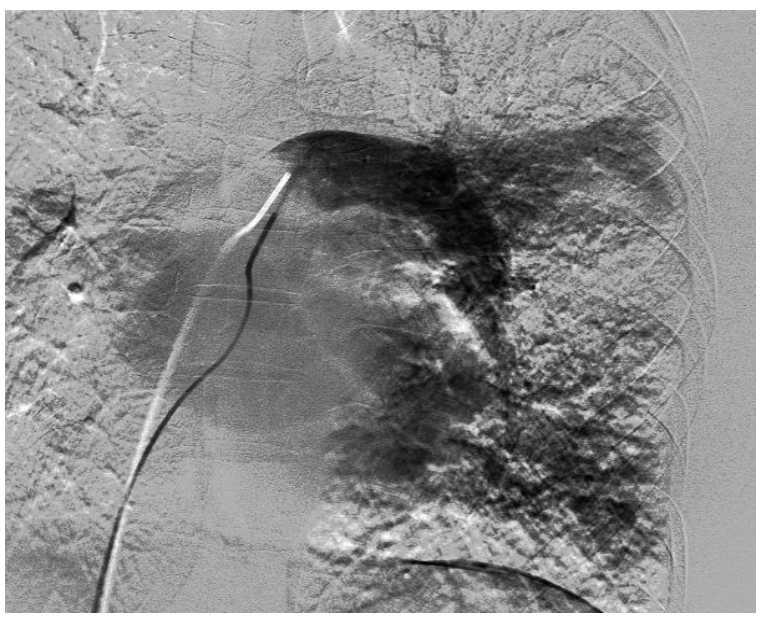

Figure 5a. DSA of selective catheterization of Left Pulmonary artery which showed diffuse PAVMs in the left lung.

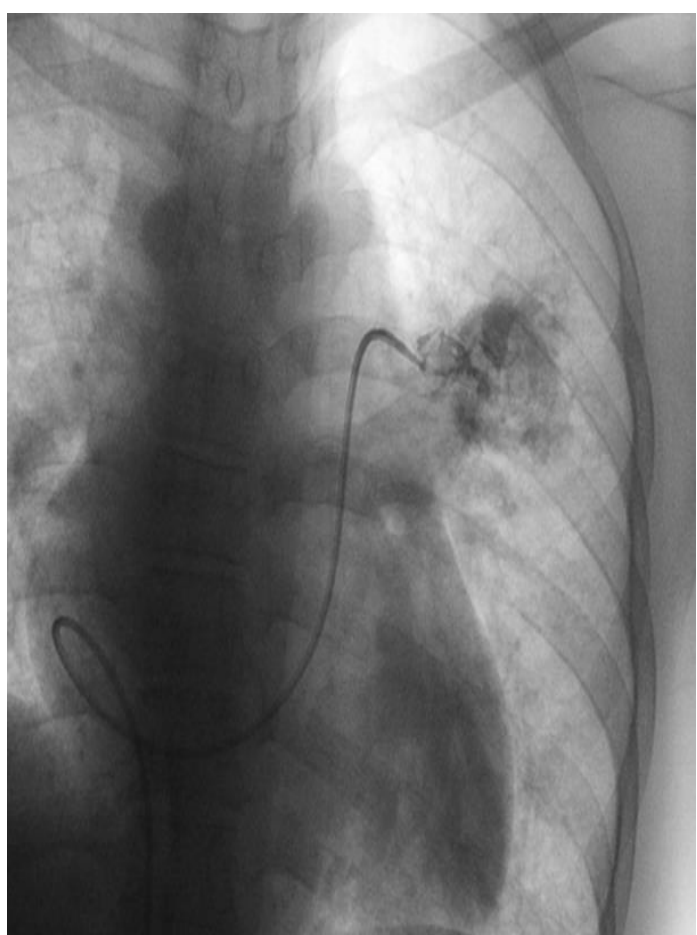

Figure $5 b$. Selective catheterization of a PAVM.

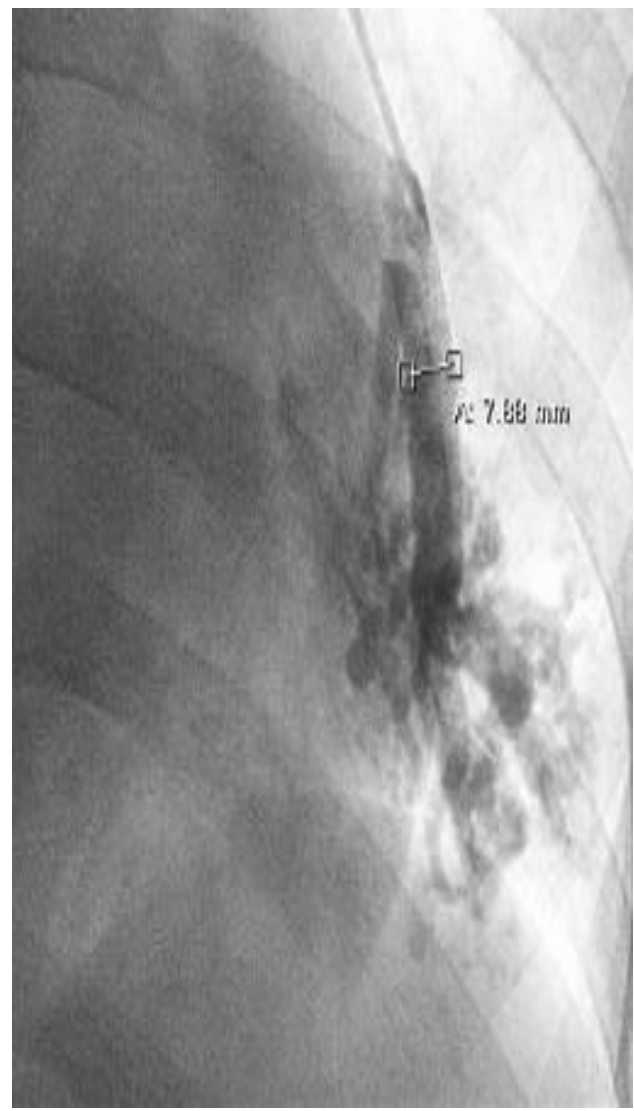

Figure 5c. Feeder artery measuring $7.8 \mathrm{~mm}$ 
Because the lesions were multiple and widespread, conservative resection was not applicable. A combined staged approach was initially considered. Staged Embolization of the right PAVM, followed by Left pneumonectomy was proposed. Although he is a viable candidate for surgery with an acceptable predicted postoperative lung function, Surgery was not an option accepted by the relatives due to its high morbidity and high mortality.

A conservative approach of transcathether embolization of the 3 large PAVMs in the left lung with the intention of reduction of the right to left shunt, improve oxygenation and lessen risk of paradoxical embolization and stroke was finally attempted. Pre- procedural arterial blood gas showed hypoxemia of pO2 of 45, saturations of $63 \%$ and baseline 6 min walk test was 250 meters.

A total of 2 Gianturco steel coils (Figure 6a) in the left upper PAVM, 2 coils in the left mid PAVM and 2 coils in the left lower basal PAVM were deployed. (Figure 6b).

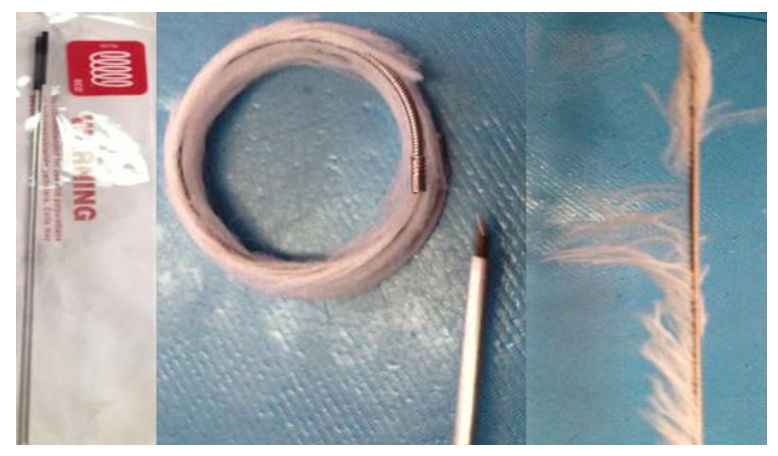

Figure 6a. Gianturco steel coils.

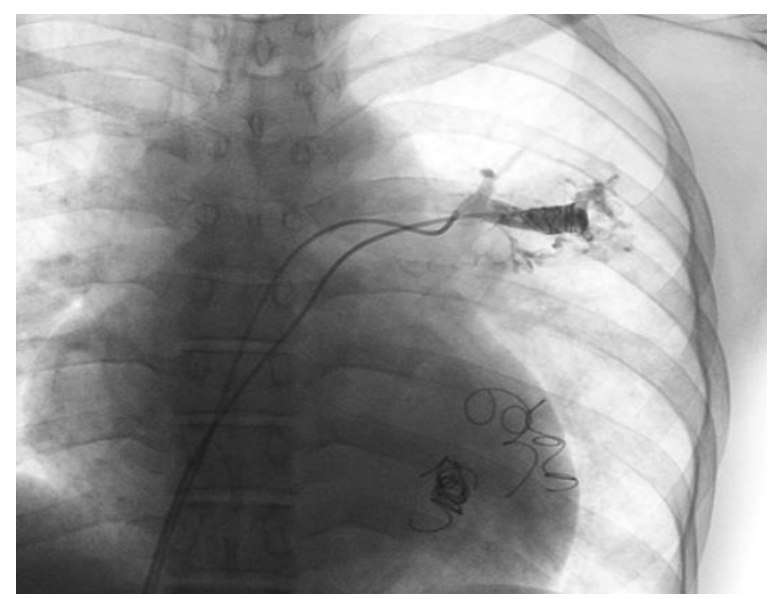

Figure 6b. Gianturco steel coils deployed at 3 large PAVMs.

Post-operatively, the patient's oxygen saturation immediately went up $98 \%$. He developed hemoptysis and presented with mild transient ischemic attacks day 2 post-op which spontaneously resolved and did not recur. He was discharged asymptomatic with oxygen saturation at room air of $86 \%$.

After 2 months, the patient came back with no recurrence of bleeding episodes and transient ischemic attacks. His oxygen saturation at room air was $80-86 \%$. His 6-minute walk test was 450 meters. A repeat pulmonary angiography for surveillance and possibly embolization of other PAVMs after several months was planned.

\section{Discussion}

Pulmonary arteriovenous malformations (PAVMs) are direct abnormal communications between pulmonary arteries and pulmonary veins. It is a rare disorder with an incidence of $2-3 / 1,000,000$ population. More than $80 \%$ of PAVMs are congenital. ${ }^{1}$ Approximately $70 \%$ of cases are associated with Osler-Weber-Rendu Disease or Hereditary Hemorrhagic Telangiectasia. ${ }^{2}$

Osler-Weber-Rendu Disease is an autosomal dominant disorder with widespread cutaneous, mucosal and visceral telangiectases. ${ }^{1} \quad$ Spontaneous recurrent epistaxis, multiple telangiectasias in typical locations, proven visceral AVM (lung, liver, brain, and spine) and a positive first degree family member forms the Curacao criteria for diagnosis of the condition. Our patient met 3 out of the 4 criteria.

The classic triad of pulmonary arteriovenous malformations (dyspnoea, cyanosis and clubbing) is present in our patient but is usually present in only $10 \%$ of the cases. $^{3}$ Usual physical examination findings are superficial telangiectasias in the lower lip, nose, mouth, chest and upper extremities, ${ }^{3}$ and a systolic bruit in the lung bases which increases with inspiration and forced inspiration with a closed glottis after full expiration (Muller maneuver). Complications, including erythrocytosis, hyperviscosity syndromes, cerebral abscess, stroke or transient ischemic attacks due to paradoxical embolization, may occur in $33 \%$ of cases. All of these were present in our patient. ${ }^{4}$

For patients suspected of PAVMs and/or Osler-WeberRendu Disease, a chest radiograph may show rounded multilobular opacities and a peripheral arterial blood gas may show $<92 \%$ or with $>4 \%$ change on moving from supine to upright position which are suggestive findings. ${ }^{2}$ Chest CT with contrast will document the presence of PAVMs with specificity of $100 \%$. Contrast echocardiography is recommended to evaluate for any concomitant intracardiac abnormality and shunting. Contrast pulmonary angiography remains the gold standard to confirm presence of PAVMs and to define the vascular architecture of pulmonary vasculature which is necessary before therapeutic embolization or surgical resection. ${ }^{1,5}$

Treatment of a PAVM is indicated in patients with progressive enlargement of the lesions, paradoxical embolization, persistent symptomatic hypoxemia and with identified feeding vessels of $3 \mathrm{~mm}$ or larger. Surgery using lung conserving resection with segmentectomy or local 
excision is the general recommendation for PAVMs but is not applicable to this patient with diffuse bilateral PAVMs. For multiple bilateral PAVMS there is no standardized treatment. At present there is no guideline on how to assess suitability of these patients for surgery. The American College of Chest Physicians 2007 Guideline on Lung Resection for Lung Cancer was applied to our patient. Baseline FEV of $>1.5 \mathrm{~L}$, >2L for pneumonectomy and DLCO of $>80 \%$ confers average risk. A Predicted Postoperative (PPO) lung function can be computed using the formula:

\section{Pre-operative FEV (1- number of functional segments to be removed)} Total number of functional lung segments

$0.8 \mathrm{~L}$ PPO FEV is the lower limit to allow patients to undergo surgical resection. ${ }^{7}$ Removing approximately 9 segments in doing a Left pneumonectomy on this patient will confer $9 \%$ operative mortality, $53-60 \%$ risk for long term disability and recurrence rate of $12 \% .^{7}$ These statistics may only apply if the remaining lung segments are normally functioning and free of disease, which is not the case for our patient.

Embolization is a suitable alternative especially for multiple PAVMs. It involves the localization of the PAVMs followed by selective catheterization of the feeding artery. An amplatzer device, steel coil or vascular plug is advanced through the catheter and placed distal to any branch of the vessel. Steel coil embolization is preferred due to its association with low morbidity and good outcome in terms of right to left shunt reduction. Treatment success rate in high volume centers is $75 \%$ with delayed recanalization and recurrence of $12-17 \%{ }^{8}$

To attempt a complete embolization of this patient's diffuse and widespread PAVMs is obviously impossible. Based on a study of Gupta et al on 66 patients with Multiple PAVMs, incomplete embolization with remaining feeders $>3$ mm can still impart significant reduction of $19 \%$ in mean Right to left shunting, with increase of $8 \%$ in arterial saturation. ${ }^{9}$ Although no apparent change in maximum workload should be expected, this patient post embolization presented with significant improvement in functional capacity.

Embolization carries inherent properties of delayed recanalization and recurrence. As recruitment of new arteriovenous beds post operatively remains a concern for this patient, repeat PA for surveillance \pm embolization is part of the long-term plan. Regular follow-up should also include pulse oximetry checks, echocardiocarphy and chest radiography.

A combined surgery and staged embolization ${ }^{10}$ and bilateral lung transplantation ${ }^{11}$ are other approaches that have been attempted on multiple bilateral PAVMs. These have showed improvement on oxygenation and functional capacity, but these cases had no long term follow-up.
To date there is no long- term survival studies concerning PAVMs and HHT. If left untreated, the approximate growth rate of these malformations is about 5$10 \mathrm{~mm}$ every $5-15$ year. The morbidity is $50 \%$ in untreated versus $3 \%$ in patients who were treated. PAVM related mortality is $1-12 \% .^{12}$

\section{Conclusion}

Presented herein is a rare case of multiple pulmonary arteriovenous malformations with bilateral lung involvement in a patient with Osler-Weber-Rendu disease. The incidence of this condition in the adult population is unknown and may be underestimated in our local setting. A high index of suspicion should be upheld in patients with chronic cyanosis, chronic history of bleeding, and brain abscess. Management of these rare diffuse intrapulmonary malformations and its associated complications remains a challenge and should be highly individualized.

\section{References}

1. Khurshid I, Downie GH. Pulmonary arteriovenous malformations: A Review. Postgrad Med J. 2002; 78(918):191-7.

2. Gossage JR, Kanj G. Pulmonary arteriovenous malformations: A State of the art review. Am J Respir Crit Care Med. 1998; 158(2):643-61.

3. Wirth JA, Pollak JS, White RIJ, et al. Pulmonary arteriovenous malformations. Curr Pulmonol Crit CareMed. 1996; 17:261-98.

4. Shovlin C, et al. Murray and Nadel's Textbook of Respiratory Medicine. 2010; Chapter 54: Med Consult.

5. van Gent MW, Post MC, Luermans JG, et al. Screening for pulmonary arteriovenousmalformations using transthoracic contrast echocardiography: a prospective study. Eur Respir J. 2009, 33:85-91.

6. Puskas JD, Allen MS, Moncure AC, et al. Pulmonary arteriovenous malformations: Therapeutic options. Ann Thorac Surg. 1993; 56:253-8.

7. Colice GL, Shafazand S, Griffin JP, et al. Physiologic evaluation of the patientwith lung cancer being considered for resectional surgery: ACCP Evidenced-Based Clinical Practice Guidelines. Chest. 2007; 132(3 Suppl):161s-177s

8. Ando K, Mochizuki A, Kurimoto N, et al. Coil embolization for pulmonary arteriovenousmalformation as an organ-sparing therapy:outcome of long-term follow-up. Ann Thorac Cardiovasc Surg. 2011;17(2);118-23.

9. Gupta P, Mordin C, Curtis J, Hughes JMB, Shovlin CL, Jackson JE. Pulmonary Arteriovenous Malformations: effect of embolization on right to left shunt, hypoxemia and exercise tolerance in 66 patients, AJR. 2002; 179:347-55.

10. Wallenhaupt S, D'Souza V. Combined radiological and surgical management of arteriovenous malformation of the lung. Ann Thorac Surg. 1988; 45:213-15.

11. Reynaud-Gaubert M, Thomas P, Gaubert JY, et al. Pulmonary arteriovenous malformations: lung transplantation as a therapeutic option. Eur Respir J. 1999; 14:1425-28.

12. Shovlin CL, LeTarte M. Hereditary hemorrhagic telangiectasia and pulmonary arteriovenous malformations: issues in clinical management and review of pathogenetic mechanims. Thorax. 1999;54;714-29. 